\title{
Therapeutic impact and routine application of next-generation sequencing: A single institute study
}

\author{
ROCÍO HERNÁNDEZ-PACHECO ACOSTA*, MARÍA DEL CARMEN DAMAS FUENTES*, \\ NICOLÁS GALLEGO PENA, PILAR SOTO ROJAS and CARMEN BEATO ZAMBRANO \\ Department of Medical Oncology, Virgen Macarena University Hospital, 41009 Seville, Spain
}

Received November 2, 2021; Accepted February 3, 2022

DOI: $10.3892 /$ br.2022.1516

\begin{abstract}
Genomic sequencing of tumor tissues provides information on actionable gene aberrations that have diagnostic and therapeutic significance and may guide clinical management through the use of targeted therapies. The indications for these techniques and their possible limitations for application in daily practice should be established as a priority. In the present study, a group of patients with few suitable therapeutic options who were eligible for a next-generation sequencing (NGS) analysis were analyzed, and the molecular targets identified and their therapeutic impact are described. A series of 26 patients treated at the Virgen Macarena Hospital for whom an NGS study was requested between January 2017 and December 2019 were reviewed. Actionable molecular alterations were identified in 20 of the cases, and 4 patients received NGS-guided treatment. NGS techniques represent a novel opportunity for guiding treatment in cancer patients. Patients with few therapeutic alternatives, either due to diagnosis, atypical evolution or resistance to standard therapy, may be suitable candidates.
\end{abstract}

\section{Introduction}

Precision medicine consists of targeted treatments based on genetic mutations, biomarkers or phenotypic characteristics that distinguish a particular patient from others with similar clinical presentations (1). These individualized therapeutic strategies first emerged with the approval of tamoxifen in breast cancer in 1971 (2). In 1998 the first drugs against target mutations, such as trastuzumab in HER 2+ breast cancer, appeared (3), and nowadays, targeted treatment is a reality

Correspondence to: Dr Rocío Hernández-Pacheco Acosta or Dr María del Carmen Damas Fuentes, Department of Medical Oncology, Virgen Macarena University Hospital, Avenida del Doctor Fedriani 3, 41009 Seville, Spain

E-mail: rociohpa@gmail.com

E-mail: carmendamasf@gmail.com

*Contributed equally

Key words: next-generation sequencing, driver mutations, tumor-agnostic treatment, cancer of unknown primary in oncology. In recent years, there has been a diagnostic revolution, thanks to increasing knowledge of the molecular biology and the development of new DNA sequencing techniques, which have led to a notable increase in the number of molecular targets identified in the last decade (4).

DNA sequencing was first described in 1977 by Maxam and Gilbert (5). Their techniques helped detect nucleotide changes and insertions or deletions in one or more genes, and is considered a milestone central to sequencing of the human genome in 2001 (6). The continuous development of these techniques led in 2005 to the emergence of mass sequencing technologies, known as next-generation sequencing (NGS), facilitating the mass, parallel reading of millions of sequences in a quick and economic manner $(7,8)$. These techniques can analyze panels of specific genes, the exome and the entire genome, and detect point mutations (insertions, deletions, base substitutions and genetic rearrangements) (9). They can also quantify the tumor mutational burden (TMB) and identify microsatellite instability (MSI), both factors associated with an immunotherapeutic response (10).

These techniques have marked a shift in the cancer treatment model towards therapeutic choices based on a specific genomic alteration, regardless of the type and location of the tumor: This concept is known as tumor-agnostic treatment (11). An example of this is the United States Food and Drink Administration (FDA) approval of pembrolizumab for patients with MSI tumors, and larotrectinib and entrectinib for tumors with NTRK fusions (11). Clinical trials have also undergone a paradigm shift, with the appearance of new models: 'umbrella' trials that include patients with tumors with the same histology, and stratify them according to genetic alterations, and 'basket' trials that select patients with tumors that share common mutations, regardless of their histology (12).

Although the contributions of NGS is undeniable, its application in clinical practice is still a challenge in oncology, primarily in terms of the development of algorithms to facilitate the analysis and interpretation of results, and the creation of guidelines and recommendations to help identify patients who might benefit from this technology $(13,14)$.

Rare cancers are defined as those with an annual incidence of less than 6/100,000 in Europe (15). Cancer of unknown primary (CUP) is defined as a metastatic cancer for which a standardized diagnostic workup fails to identify the primary origin (16). Both rare cancers and CUP pose challenges for 
diagnosis, treatment and clinical decision-making. Clinical trials in tumors of this type are also scarce and it is hard to build up new knowledge and expertise.

In the present study, the characteristics of a group of cancer patients who underwent an NGS study were described, analyzing the molecular alterations identified and their translation to therapeutic alternatives. The aim of this study was to show the therapeutic impact of these techniques in a population of rare cancers, CUP, and other tumors that were difficult to manage therapeutically, due to either atypical progress or resistance to standard treatments.

\section{Patients and methods}

Patients and sequencing. A series of 26 patients in whom an NGS study was requested at the Virgen Macarena Hospital in Seville between January 2017 and December 2019 were retrospectively reviewed.

Male or female patients $>18$ years with a histopathological diagnosis of carcinoma with metastatic disease were included. The patients included 14 males and 12 females. The age range of the patients was 29-82 years old, with a median age of 60. Patient clinical records were requested from the hospital's records department, and data were collected by the investigators.

Patients underwent NGS testing using FoundationOne ${ }^{\circledR}$ CDx (F1CDx, Foundation Medicine, Inc.) a single commercially available platform. The criterion for requesting the study was the subjective perception of benefit in patients with limited treatment options, as determined by the oncologist. Comprehensive genomic analyses were performed using these FoundationOne ${ }^{\circledast} \mathrm{CDx}$ tests, provided in the context of an expanded access program with a research purpose.

F1CDx is performed exclusively as a laboratory service using DNA extracted from formalin-fixed paraffin-embedded tumor samples, 50-200 ng of which undergoes whole-genome shotgun library construction and hybridization-based capture of 4,557 exons from 287 cancer-related genes and 47 introns from 19 genes frequently rearranged in solid tumors. Hybrid capture libraries are sequenced to high depth using the Illumina HiSeq2000 platform (Illumina, Inc.). Sequence data are processed using a customized analysis pipeline designed to accurately detect multiple classes of genomic alterations including base substitutions, short insertions/deletions, copy-number alterations, and selected gene fusions (17).

The F1CDx-targeted NGS was the first FDA-approved tissue-based platform validated for all solid tumors (18). It applies NGS across 324 known solid tumor driver genes with high accuracy. Both TMB and MSI status were also assessed.

The following variables were collected: Age, sex, tumor histology, previous lines of treatment, molecular alterations, presence of MSI and TMB, and NGS-guided therapies received.

This study was a retrospective, non-interventional study, and adhered to the 1964 Helsinki Declaration of Ethical Principles for Medical Research Involving Human Subjects, and the need for approval was not required by the hospital given its retrospective nature. Informed consent was obtained from all subjects involved included in the present study.
Table I. Demographics and clinical characteristics of the patients.

$\begin{array}{lr}\text { Age, median (interquartile range) } & 60(52-72) \\ \text { Sex, } \mathrm{n}(\%) & 14(53.8) \\ \text { Male } & 12(46.2) \\ \text { Female } & \\ \text { Tumor type, } \mathrm{n}(\%) & 11(42.3) \\ \text { Cancer of unknown primary } & 6(23.1) \\ \text { Cancer progression after several lines of } & \\ \text { treatment } & 5(19.2) \\ \text { Cancer with atypical evolution } & 4(15.4) \\ \text { Low-incidence tumors } & \\ \text { Previous treatment lines, } \mathrm{n}(\%) & 11(42.3) \\ 0 & 4(15.4) \\ 1 & 11(42.3) \\ \geq 2 & \end{array}$

Statistical analysis. A descriptive analysis of demographic, clinical, molecular and therapeutic data was generated. Data are presented as absolute values and proportions in the case of discrete variables, and as the mean \pm standard deviation (SD) or median and interquartile range (IQR) for continuous variables. Statistical analysis was performed using SPSS version 20.0.0 (IBM Corp.).

\section{Results}

The results of the 26 patients who underwent an NGS study between January 2017 and December 2019 are discussed. Clinical and demographic characteristics, tumor histology, and previous lines of treatment are listed in Table I. Among the cases with progressive disease despite multiple treatment lines, 3 patients had colorectal carcinoma, 2 had gynecological disease, and 1 had prostate carcinoma. The group of tumors of atypical evolution included 1 patient with a diagnosis of triple-negative breast carcinoma, 2 with BRAF wild-type melanoma, 1 with pancreatic carcinoma and 1 with gastric adenocarcinoma. The group of patients with low incidence tumors included 1 patient with renal papillary carcinoma, 1 with squamous cell carcinoma of the penis, 1 with nasopharyngeal carcinoma and 1 with adrenal cortical carcinoma.

Actionable molecular alterations were found in most of the cases analyzed $(\mathrm{n}=20,76.9 \%)$, as shown in Table II. Mutational burden was studied, identifying $46.1 \%$ low mutational burden (TMB-low) in $46.1 \%$ of the cases, $15.4 \%$ intermediate (TMB-Intermediate), and 3.8\% high (TMB-high). In the rest of the cases (34.6\%), the mutational load could not be determined. Similarly, the presence of MSI was analyzed, identifying high MSI (MSI-high) in 1 case (3.8\%).

Among the 26 selected patients, 4 (15.4\%) benefited from NGS-guided treatment, as shown in Table III. Of the 11 patients with carcinoma of unknown primary (CUP), actionable alterations were found in 7 patients, of which 2 received NGS-guided treatment with a tumor-agnostic indication. 
Table II. Molecular alterations identified by next-generation sequencing according to histological subtype.

\begin{tabular}{cl}
\hline Histological diagnosis & \multicolumn{1}{c}{ Actionable molecular alterations } \\
\hline Cancer of unknown primary: Patient no. & ATM Q1098 \\
1 & KDR, KIT and PDGFRA amplifications \\
2 & CD274 (PD-L1) amplification, PTEN loss \\
3 & ERBB3, FBXW7 Y86fs*3, R465C, PTEN K267fs*9, TMB-High, \\
& IMS-High \\
4 & ERBB2 Q709L \\
5 & ATM Q1970 \\
6 & PTEN R130G \\
7 & ROS1 G1196R \\
8 & None \\
9 & None \\
10 & None \\
11 & None
\end{tabular}

Pancreatic adenocarcinoma: Patient no.

12

Melanoma: Patient no.

13

14

Adrenocortical carcinoma: Patient no.

15

Colon adenocarcinoma: Patient no.

16

17

Rectal adenocarcinoma

18

Papillary renal carcinoma

19

Gastric adenocarcinoma

20

Ovarian endometrioid adenocarcinoma

21

Penile squamous cell carcinoma

22

Undifferentiated nasopharyngeal carcinoma

23

Ovarian serous carcinoma

24

Prostate adenocarcinoma

25

Breast carcinoma

26
ATM R2993

BRAF V600E

None

NF1 E169

KRAS

KRAS G12V, PALB2

KRAS G13D

None

AKT1 E17K

KRAS G12A, PTEN A126T

CCND1, CD274 (PD-L1) and PDCD1LG2 (PD-L2) amplifications

KRAS G12C, RAF1 amplification

None

Androgen receptor amplification

NF1 Q1174

\section{Discussion}

In this study, actionable mutations were identified in 20 of the 26 cases analyzed, and 4 patients were able to benefit from targeted therapy based on the results of genomic sequencing. Clinical genetics is a field that is currently developing exponentially, so in coming years it can be expected to see an increase in the number of cases in which actionable alterations that can be associated with a targeted therapy are found. Thus, the priority should be to identify the cases that may benefit most from these techniques.

Correct interpretation of the biomarkers or genetic variations revealed by sequencing is critical in precision medicine. Although there is an increase in the identification 
Table III. Next-generation sequencing-guided targeted therapy.

\begin{tabular}{llll}
\hline Histological diagnosis (patient no.) & \multicolumn{1}{c}{$\begin{array}{c}\text { Actionable } \\
\text { molecular alterations }\end{array}$} & Targeted treatment & Indication \\
\hline Cancer of unknown primary (Patient 4) & TMB-high & Pembrolizumab & $\begin{array}{l}\text { Tumor-agnostic } \\
\text { indication }\end{array}$ \\
& MSI-high & & \\
& ERBB3 & & \\
& FBW7 Y86fs*3, R465C & & \\
Cancer of unknown primary (Patient 5) & EREN K267s*9 & Trastuzumab & Compassionate use \\
Gastric adenocarcinoma (Patient 20) & AKT1 E17K & Everolimus & Approved on indication \\
Ovarian endometrioid carcinoma (Patient 21) & KRAS G12A & Everolimus & Compassionate use \\
& PTEN A126T & &
\end{tabular}

of mutational biomarkers with an associated targeted therapy, the detection of these mutations alone is insufficient, and correct interpretation by specialists is essential to determine the clinical implications for the best therapeutic approach (19). Not all genetic alterations are clinically actionable, hence the importance of creating guidelines or recommendations to aid decision-making in clinical practice.

Advances in sequencing techniques have led us to question not their usefulness, but rather their correct indication; in which patients and in what type of tumors might these techniques be of value? And at what point in the disease history should they be applied? Based on current evidence, the percentage of patients with metastatic cancer eligible for NGS has increased from 6\% in 2006 to $8.33 \%$ in 2018 (19). It is estimated that in $\sim 5 \%$ of patients, a clinical benefit from the application of NGS may be obtained (20). In the present study, actionable mutations were found in up to $77 \%$ of the cases, and $15.4 \%$ of the patients received NGS-guided treatment.

Several authors have taken different positions on the implementation of NGS techniques in oncology practice. Proposed indications include patients with advanced-stage cancer in whom a first-line decision depends on multiple molecular markers (21) or patients with rare tumors (sarcomas, mesotheliomas, cholangiocarcinoma or CUP), that due to their low incidence are not usually well represented in phase III clinical trials and lack options for standard second-line therapies (22). Performing NGS in these patients could offer them access to treatments or clinical trials.

A recent review of 10 published studies using NGS in CUP patients identified mutations with potential therapeutic relevance in $30-85 \%$ of cases (23). Similarly, in the present study, $63.6 \%$ of the patients with CUP presented actionable molecular alterations and 18\% received NGS-guided treatment.

NGS is changing mindsets in the treatment of cancer patients, paving the way towards novel concepts such as tumor-agnostic treatment. In 2017, the FDA approved the first tumor-agnostic treatment; pembrolizumab in patients with MSI-high solid tumors (11). Its indications have recently been expanded, and in 2020, the FDA also approved its use in
TMB-high solid tumors (24). This approach is supported by the findings of the KEYNOTE-158 clinical trial (25), which looked at 790 patients and up to 10 different types of tumors. A total of $102(13 \%)$ of the study patients had TMB-high disease, and of these, $29 \%$ responded to pembrolizumab treatment, compared to only $6 \%$ of the patients without TMB-high disease. These results show that the mutational burden selects a subgroup of patients who may have a robust and long-lasting tumor response to pembrolizumab monotherapy, suggesting that TMB could be a useful biomarker in this regard (25). Similarly, in the present study, the patient in whom TMB-high disease was detected received targeted therapy with pembrolizumab, in line with that described in the literature.

The main limitation of the present study is the small sample size. However, recording the modest scale of NGS analysis can be informative for the members of the oncology community, especially if it is supported and compared with similar data published in the literature. A series of 26 cases in which an NGS study was requested at the Virgen Macarena Hospital in Seville, an 850-bed community hospital with an assigned population of 480,000 users in Western Andalusia, staffed by over 5,000 professionals, was analyzed is presented in the present study.

Actionable gene alterations were identified in $76.9 \%$ of the cases, and $15.4 \%$ of the patients received genotype-matched therapy. A larger study conducted in Japan, which examined 230 cases of advanced solid tumors (comprised of $>30$ tumor types), reported actionable genetic aberrations in 59.4\% of the cases (26). Despite the sample size limitation of the present study, a similar distribution was observed in both the present study and the previous study; $13.4 \%$ of the patients received genotype-matched therapy in the Japanese study compared to $15.4 \%$ in the present study (26). These results indicate the feasibility and usefulness of gene panel testing in a clinical oncology setting, with the remaining challenge of identifying patients who will benefit from such studies.

To date, there remains a lack of a useful set of guidelines for the planned and controlled implementation of NGS in clinical practice. An international group of experts from the European Society for Medical Oncology (ESMO) has recently published the first recommendations on the use of 
NGS techniques in patients with metastatic tumors (27). These recommendations have been developed on the basis of the Molecular Objectives Clinical Action Scale (ESCAT) which has 6 levels of clinical evidence for molecular alterations in cancer (28), ranked from mutations with unequivocal clinical evidence (level I) to mutations with still unknown relevance (level X). For each tumor type, the ESMO investigators used the ESCAT classification and the prevalence of abnormalities to estimate the number of cases that would need to be evaluated with NGS to find 1 patient who could be treated effectively with a given drug.

ESMO has proposed 3 levels of recommendations for the use of NGS: recommendations for daily practice with repercussions on public health (ESCAT level I); recommendations for research; and patient-centered recommendations. It also recommends the routine use of NGS in all patients with metastatic non-squamous non-small cell lung cancer, prostate cancer, ovarian cancer and cholangiocarcinoma (28). Specific recommendations are proposed for other types of cancers. In CUP, genetic sequencing may also be used despite the lack of level I evidence (29).

Although the usefulness of novel sequencing techniques in oncology is undeniable, questions remain: Should NGS be standardized in routine clinical practice? Which patients would benefit most from an NGS study? Are oncologists equipped to interpret the results? The results are clearly encouraging, but an increased understanding is required of the exact benefits of these new techniques, and further studies are needed to substantiate these findings. This modest but informative study underscores the clinical utility of NGS in patients with few therapeutic alternatives.

Although the profile of patients that may benefit most from NGS, and in whom its application would be more cost-effective, is not known, the present study showed that patients with few therapeutic alternatives, either due to diagnosis, atypical evolution or resistance to standard therapy, may be suitable candidates for such studies.

\section{Acknowledgements}

Not applicable.

\section{Funding}

No funding was received.

\section{Availability of data and materials}

The datasets used and/or analyzed during the present study are available from the corresponding authors on reasonable request.

\section{Authors' contributions}

CBZ contributed to the conception and design of the study, and to patient recruitment. NGP, PSR contributed to data acquisition. MDCDF and RHPA contributed to data interpretation and writing of the manuscript. All authors reviewed and approved the final manuscript. MDCDF and RHPA confirm the authenticity of all the raw data.

\section{Ethics approval and consent to participate}

This study was a retrospective, non-interventional study, and adhered to the 1964 Helsinki Declaration of Ethical Principles for Medical Research Involving Human Subjects, and the need for approval was not required by the hospital given its retrospective nature. Informed consent was obtained from all subjects involved included in the present study.

\section{Patient consent for publication}

Not applicable.

\section{Competing interests}

The authors declare that they have no competing interests.

\section{References}

1. Jameson JL and Longo DL: Precision medicine-personalized, problematic, and promising. N Engl J Med 372: 2229-2234, 2015.

2. Jordan VC: Fourteenth gaddum memorial lecture. A current view of tamoxifen for the treatment and prevention of breast cancer. $\mathrm{Br}$ J Pharmacol 110: 507-517, 1993.

3. Buchdunger E, Zimmermann J, Mett H, Meyer T, Müller M, Druker BJ and Lydon NB: Inhibition of the Abl protein-tyrosine kinase in vitro and in vivo by a 2-phenylaminopyrimidine derivative. Cancer Res 56: 100-104, 1996.

4. Andre F, Mardis E, Salm M, Soria JC, Siu LL and Swanton C: Prioritizing targets for precision cancer medicine. Ann Oncol 25: 2295-2303, 2014

5. Maxam MA and Gilbert W: A new method for sequencing DNA. Proc Natl Acad Sci USA 74: 560-564, 1977.

6. Heather JM and Chain B: The sequence of sequencers: The history of sequencing DNA. Genomics 107: 1-8, 2016.

7. Mardis ER: Next-generation DNA sequencing methods. Annu Rev Genomics Hum Genet 9: 387-402, 2008.

8. Shendure $\mathrm{J}$ and Ji H: Next-generation DNA sequencing. Nat Biotechnol 26: 1135-1145, 2008.

9. Behjati S and Tarpey PS: What is next generation sequencing? Arch Dis Child Educ Pract Ed 98: 236-238, 2013.

10. Marabelle A, Le DT, Ascierto PA, Di Giacomo AM, De Jesus-Acosta A, Delord JP, Geva R, Gottfried M, Penel N, Hansen AR, et al: Efficacy of pembrolizumab in patients with noncolorectal high microsatellite instability/mismatch repair-deficient cancer: Results from the phase II KEYNOTE-158 study. J Clin Oncol 38: 1-10, 2020.

11. Looney AM, Nawaz K and Webster RM: Tumour-agnostic therapies. Nat Rev Drug Discov 19: 383-384, 2020.

12. Park JJH, Hsu G, Siden EG, Thorlund K and Mills EJ: An overview of precision oncology basket and umbrella trials for clinicians. CA Cancer J Clin 70: 125-137, 2020.

13. Goodwin S, McPherson JD and McCombie WR: Coming of age: Ten years of next-generation sequencing technologies. Nat Rev Genet 17: 333-351, 2016.

14. Liu Z, Zhu L, Roberts R and Tong W: Toward clinical implementation of next-generation sequencing-based genetic testing in rare diseases: Where are we? Trends Genet 35: 852-867, 2019.

15. Gatta G, Capocaccia R, Botta L, Mallone S, De Angelis R, Ardanaz E, Comber H, Dimitrova N, Leinonen MK, Siesling S, et al: Burden and centralised treatment in Europe of rare tumours: Results of RARECAREnet-a population-based study. Lancet Oncol 18: 1022-1039, 2017.

16. Subbiah IM, Tsimberidou A, Subbiah V, Janku F, Roy-Chowdhuri S and Hong DS: Next generation sequencing of carcinoma of unknown primary reveals novel combinatorial strategies in a heterogeneous mutational landscape. Oncoscience 4: 47-56, 2017.

17. Frampton GM, Fichtenholtz A, Otto GA, Wang K, Downing SR, He J, Schnall-Levin M, White J, Sanford EM, An P, et al: Development and validation of a clinical cancer genomic profiling test based on massively parallel DNA sequencing. Nat Biotechnol 31: 1023-1031, 2013. 
18. Alegretti M, Fabi A, Buglioni S, Martayan A, Conti L, Pescarmona E, Ciliberto $G$ and Giacomini P: Tearing down the walls: FDA approves next generation sequencing (NGS) assays for actionable cancer genomic aberrations. J Exp Clin Cancer Res 37: 47, 2018.

19. Matthijs G, Souche E, Alders M, Corveleyn A, Eck S, Feenstra I, Race V, Sistermans E, Sturm M, Weiss M, et al: Guidelines for diagnostic next-generation sequencing. Eur J Hum Genet 24 $1515,2016$.

20. Marquart J, Chen EY and Prasad V: Estimation of the percentage of US patients with cancer who benefit from genome-driven oncology. JAMA Oncol 4: 1093-1098, 2018.

21. Schwartzberg L, Kim ES, Liu D and Schrag D: Precision oncology: Who, how, what, when, and when not? Am Soc Clin Oncol Educ Book 37: 160-169, 2017.

22. Colomer R, Mondejar R, Romero-Laorden N, Alfranca A, Sanchez-Madrid F and Quintela-Fandino M: When should we order a next generation sequencing test in a patient with cancer? EClinicalMedicine 25: 100487, 2020.

23. Binder C, Matthes KL, Korol D, Rohrmann S and Moch H: Cancer of unknown primary-Epidemiological trends and relevance of comprehensive genomic profiling. Cancer Med 7: 4814-4824, 2018.

24. Subbiah V, Solit DB, Chan TA and Kurzrock R: The FDA approval of pembrolizumab for adult and pediatric patients with tumor mutational burden $(\mathrm{TMB}) \geq 10$ : A decision centered on empowering patients and their physicians. Ann Oncol 31: $1115-1118,2020$

25. Marabelle A, Fakih M, Lopez J, Shah M, Shapira-Frommer R, Nakagawa K, Chung HC, Kindler HL, Lopez-Martin JA, Miller WH Jr, et al: Association of tumour mutational burden with outcomes in patients with advanced solid tumours treated with pembrolizumab: Prospective biomarker analysis of the multicohort, open-label, phase 2 KEYNOTE-158 study. Lancet Oncol 21: 1353-1365, 2020 .
26. Sunami K, Ichikawa $H$, Kubo T, Kato M, Fujiwara $Y$, Shimomura A, Koyama T, Kakishima H, Kitami M, Matsushita $\mathrm{H}$, et al: Feasibility and utility of a panel testing for 114 cancer-associated genes in a clinical setting: A hospital-based study. Cancer Sci 110: 1480-1490, 2019.

27. Mosele F, Remon J, Mateo J, Westphalen CB, Barlesi F, Lolkema MP, Normanno N, Scarpa A, Robson M, Meric-Bernstam F, et al: Recommendations for the use of next-generation sequencing (NGS) for patients with metastatic cancers: A report from the ESMO precision medicine working group. Ann Oncol 31: 1491-1505, 2020.

28. Mateo J, Chakravarty D, Dienstmann R, Jezdic S, Gonzalez-Perez A, Lopez-Bigas N, Ng CKY, Bedard PL, Tortora G, Douillard JY, et al: A framework to rank genomic alterations as targets for cancer precision medicine: The ESMO scale for clinical actionability of molecular targets (ESCAT). Ann Oncol 29: 1895-1902, 2018.

29. Hotta K, Aoe K, Kozuki T, Ohashi K, Ninomiya K, Ichihara E, Kubo T, Ninomiya T, Chikamori K, Harada D, et al: A phase II study of trastuzumab emtansine in HER2-positive non-small cell lung cancer. J Thorac Oncol 13: 273-279, 2018.

This work is licensed under a Creative Commons Attribution-NonCommercial-NoDerivatives 4.0 International (CC BY-NC-ND 4.0) License. 